\title{
Valoración de la tutoría en el devenir de la formación universitaria
}

\section{Assessment of Mentoring Institutional Program in University Education}

\author{
Martha Patricia Astudillo Torres, Florlenis Chévez Ponce \\ Universidad Autónoma de Chiapas, Mexico; Universidad de Costa Rica, Costa Rica \\ Patricia.astudillo@unach.mx; florlenis.chevez.ponce@mep.go.cr \\ https://orcid.org/0000-0002-9013-6467; https://orcid.org/0000-0002-6295-9052
}

\section{Resumen}

El documento presenta los resultados de una
investigación en curso, que analiza la implementación
de un programa institucional de tutoría de viva voz
de los participantes, centrándose en la experiencia
del tutor y el tutorado en el devenir del proceso
educativo en un programa académico universitario.
Metodológicamente se fundamenta en el estudio
de casos, desde la perspectiva interpretativa. Los
principales hallazgos señalan que, aunque la tutoría
ha beneficiado a los estudiantes en su transitar por el
programa educativo, existen problemáticas que no son
resueltas de manera efectiva con su implementación,
tal es el caso de las deficiencias académicas que el
alumnado trae consigo desde un nivel educativo
anterior, aunada a la vulnerable situación económica
de los estudiantes de la región, lo que deviene en
deserción escolar.

\section{Palabras Clave}

Deserción escolar; Educación superior; Estudio de casos; Programa de Acción Tutorial (PAT); Tutoría

\begin{abstract}
This paper shows the results of a current research project which analyzes the implementing of a mentoring institutional program based on real time conversation between the participants, focused on the experience of the mentor and the student on the educative process of a university academic program. The methodological design is based on the premises of case study, founded on interpretative perspective. Despite mentoring benefits students coursing the educative program, main findings point towards not solved problems in an effective way with its implementation, as in the case of previous academic lacks affecting students, plus their fragile economic position characteristic of the region they come from, all of which promote dropping out the school.
\end{abstract}

\section{Keywords}

Dropping out the School; Higher Education;

Case Study; Tutorial Action Program; Mentoring

\section{Introducción}

La tutoría en el sistema de educación superior es un proceso que se ha realizado, simultáneamente, desde el surgimiento de la educación institucionalizada. Así lo demuestran las actividades tutoriales llevadas a cabo en las primeras universidades medievales, incluso, como una forma privilegiada de la docencia (Narro y Arredondo, 2013) y los diversos modelos tutoriales desarrollados e implementados 
a lo largo de varias décadas en universidades de Estados Unidos, Canadá, Inglaterra, Australia, España y México.

En el caso de la educación superior mexicana, la tutoría se ha integrado en el proceso de enseñanza y aprendizaje con la finalidad de elevar la calidad educativa y contrarrestar los problemas de deserción y rezago escolar. En este sentido, la carrera que contextualiza este estudio, la Lic. en Sistemas Computacionales de la Universidad Autónoma de Chiapas, México, presenta una elevada deserción escolar, puesto que su matrícula ha venido disminuyendo de forma progresiva del año 2010 al 2015; de tal suerte que, de contar con una matrícula de 739 estudiantes en el semestre agosto-diciembre de 2010 (UNACH, 2010), para el periodo agosto-diciembre de 2015 se contaba con 422 estudiantes (UNACH, 2015), lo que representa un decremento significativo en su población escolar.

A la vista de lo anterior, se hace necesario analizar el programa institucional de tutoría implementado en el referido centro universitario, y conocer los significados que los participantes le atribuyen a la tutoría, enfatizando la comprensión entre los tutores y los tutorados.

\section{Marco teórico}

La tutoría se define como la intervención docente en el proceso educativo de carácter intencionado. Consiste en el acompañamiento cercano al estudiante de una forma sistemática y permanente para apoyarlo y facilitarle la construcción de aprendizajes cognitivos, afectivos, socioculturales y existenciales. Implica procesos de comunicación por parte del profesorado, una atención personalizada a los estudiantes, basado en el conocimiento de sus problemas, de sus necesidades y de sus intereses específicos (Narro y Arredondo, 2013).

De igual modo, la tutoría se considera una modalidad académica que comprende acciones educativas centradas en los estudiantes: es un apoyo para el cumplimiento curricular, en el que el tutor proporciona atención educativa al alumno, orientándole para cumplir sus metas educativas. La acción tutorial busca potenciar el desempeño de los tutorados para alcanzar el perfil profesional requerido por la sociedad, son parte complementaria de la docencia de grupo y se realiza en distintos espacios y tiempos en los programas educativos. Igualmente, se constituye como un proceso continuo de inserción del alumno en el medio académico a fin de proporcionar su progreso satisfactorio en los estudios durante su formación profesional (Alvarado y Romero, 2006).

De acuerdo con estos autores, la tutoría permite conocer diversas maneras de resolver los problemas que surgen en el contexto escolar, comprender las características del plan de estudios y las opciones de trayectoria, adquirir técnicas adecuadas de lectura y comprensión y desarrollar estrategias de 
estudios. Al respecto, Herrera (2011) señala que el objetivo de la tutoría es promover el desarrollo integral de los estudiantes y es vista como un componente intrínseco de la enseñanza.

En este aspecto, la Organización de las Naciones Unidas para la Educación, la Ciencia y la Cultura (UNESCO, 1998) manifiesta que la tutoría comprende un conjunto de actividades que propician situaciones de aprendizaje y apoyan el correcto desarrollo del proceso académico, personal y profesional, al orientar y motivar a los estudiantes para que a su vez avancen y concluyan eficazmente su proceso académico; asimismo, la ANUIES (2000) señala que la tutoría es un acompañamiento personal y académico a lo largo del proceso educativo para mejorar el rendimiento académico de los estudiante, así como facilitar la solución de sus problemas escolares y desarrollar hábitos de estudio, de trabajo, de reflexión y de convivencia social.

A efectos de esta investigación, se define a la tutoría como un conjunto de actividades y acciones organizadas quebuscan mejorarel proceso educativo delos estudiantes del nivel superior, considerando sus ámbitos cognitivo y emocional. Para ello, se desarrollan estrategias que ayuden al alumnado a familiarizarse con el entorno educativo, mejorar el aprendizaje, favorecer las relaciones sociales entre pares y con el profesorado, orientar sus inquietudes profesionales, informar sobre temas de salud tanto física como mental y, en general, facilitar su transición en la institución durante su formación. Cabe mencionar que en dicha definición se toman en cuenta los elementos esenciales propuestos por los entes antes referenciados, en cuanto a tener presente un concepto de tutorías que responda a las características particulares de la población que participa en los procesos de enseñanza y aprendizaje.

Contamos con investigaciones sobre la implementación de la tutoría en el contexto educativo superior, que indagan sobre la parte cualitativa de la tutoría y los elementos indispensables para el desarrollo de un proceso tutorial de calidad, atendiendo a los tres actores principales: alumnos, docentes e institución (Alvarado y Romero, 2006); la comprensión de la visión que poseen los estudiantes de los primeros cursos de grado acerca de la tutoría en la Universidad (Rodríguez, Calvo y Haya, 2014); el análisis del programa de acción tutorial, su visión, su uso y sus resultados considerando la opinión de los profesores y los estudiantes (Alcolea y Pérez, 2012); y el estudio del programa de tutoría y sus procesos a partir de una reflexión pedagógica desde el punto de vista del profesorado (Rodríguez y Sierra, 2014).

De igual forma, las investigaciones abordan las labores de tutoría como una manera de fortalecer la docencia universitaria (Narro y Arredondo, 2013) y las estrategias de intervención educativa como apoyo para reducir los índices de reprobación y deserción escolar con base en el programa institucional de tutorías, dentro de las tendencias educativas para la educación superior (Hernández, Martínez y Carranza, 2013). 


\subsection{Abordaje de las tutorías en la educación superior}

La perspectiva teórica de las investigaciones consultadas alude a la explicación de conceptos, en la mayoría de los casos, y la presentación del estado del arte, en una minoría; únicamente un estudio se fundamenta desde la base de la teoría de los campos de producción simbólica, la teoría del habitus, algunos aportes conceptuales y teóricos para la formación en investigación. Lo anterior, pone de manifiesto el vacío que existe en la mayor parte de los estudios revisados, puesto que carecen de una justificación epistemológica que los circunscriba con la perspectiva ontológica y metodológica que deberían poseer las investigaciones educativas, en tanto que estas perspectivas aportan a los estudios cómo conciben el hecho estudiado por parte de los investigadores, así como la realidad en la que se lleva a cabo la actividad, el abordaje metodológico que se requiere para la implementación y cómo se plantea la producción del conocimiento.

Por otro lado, el sustento metodológico se corresponde con la perspectiva cualitativa, apoyada por técnicas de recolección de datos, como la entrevista en profundidad; la encuesta abierta y semiestructurada; el grupo focal; y la observación participante.

De este modo, Alvarado y Romero (2006) revelan que los aspectos cualitativos no percibidos en el trabajo tutorial universitario, corresponden al reconocimiento y la atención de necesidades particulares en tres ámbitos: la institución, la docencia y el alumno. Respecto de la institución, se deben construir los procesos de gestión para el desarrollo de los planes de tutoría de atención individual y grupal; en relación con la docencia, debe contar con los medios necesarios para la función de tutor; mientras que el alumno debe contar con el apoyo de un tutor que le brinde una asistencia acorde con las necesidades de formación.

Complementariamente se debe resaltar la función de la tutoría en relación con la reciente revaloración de la cultura juvenil, y la necesidad de tratar a los estudiantes como jóvenes en la perspectiva de una educación integral, no únicamente en el plano cognitivo sino en todas sus dimensiones como persona (Narro y Arredondo, 2013).

Por su parte, Rodríguez, Calvo y Haya (2014) señalan que los principales problemas en la implantación de un modelo tutorial consisten en las debilidades que presenta la orientación académica de los estudiantes en la transición del bachillerato a la universidad; la necesidad de integrarla en el aula; y su utilización en la mejora de la evaluación docente. En este sentido, resulta necesaria la elaboración de una guía de trabajo para la acción tutorial regenerada, revalorizada, desempolvada y sin exceso de burocracia para que sea de real utilidad (Alcolea y Pérez, 2012). Igualmente, los investigadores declaran que el alumnado considera las tutorías voluntarias muy útiles y que el profesorado debe ser provisto de documentos de orientación y herramientas para realizar esta función. 
En congruencia con lo planteado, el profesorado expresa que, aunque no existen criterios unificados de cómo llevar a cabo la tutoría académica, la acción tutorial es de suma importancia. Por ello, es necesario contar con políticas claras a nivel institucional y con un plan de formación de docentestutores que contemple una orientación respecto a la función del tutor académico, sus límites y alternativas pedagógicas, que faciliten la acción tutorial (Rodríguez y Sierra, 2014).

Al respecto, Torres (2012) manifiesta que el tutor desempeña la mediación humana que hace posible la socialización, interiorización y recreación del oficio de investigador, de manera asociada a un proceso de acompañamiento-formación cercano, estrecho y cara a cara en cada una de las tareas que comprende la actividad de investigación. En dicho acompañamiento se socializa cierta tradición científica de conocimientos y se promueve el desarrollo e interiorización de aquellos habitus científicos solicitados para ingresar como agente legítimo a un campo de producción cultural de conocimiento: el de la investigación educativa.

La implementación de programas tutoriales en las instituciones de educación superior ha favorecido el proceso de enseñanza aprendizaje, particularmente en la disminución de los índices de reprobación y deserción escolar, tal como lo manifiesta la investigación realizada por Hernández, Martínez y Carranza (2013). En ella se señala que a partir de los cursos remediales impartidos y el programa de asesoría apoyados de manera transversal por el programa institucional de tutorías, se disminuyó de un 26\% a un 22.72\% en el índice de reprobación de la matrícula total de un semestre del año 2011.

\subsection{Fundamentación epistemológica de las tutorías en la educación}

Dentro de los supuestos de la teoría histórico cultural, conocida como paradigma del constructivismo sociocultural, en occidente se fundamenta la importancia de la mediación en el potencial que puede llegar a desarrollar un individuo que lleva a cabo sus procesos cognitivos con la guía de otro individuo con más competencia; acción reconocida como Zona de Desarrollo Próximo (ZDP), elemento en el que se fundamenta el proceso de tutoría de esta investigación.

El concepto Zona de Desarrollo Próximo aplicado al campo de la educación, explica que el aprendizaje no se produce en una situación de aislamiento, sino en una situación de colaboración entre un estudiante y un adulto o un compañero más competente. La Zona de Desarrollo Próximo (ZDP) de un estudiante se define como la distancia que existe entre su nivel de resolución de problemas cuando la realiza de forma individual y cuando recibe la guía o la facilitación de otra persona más competente (Kozulin, 2000), tal como se lleva a cabo en el proceso de tutoría en las instituciones educativas.

El paradigma sociocultural se desarrolló en 1920 por Lev Vigotsky; sus presupuestos señalan que no es posible el estudio de los procesos de desarrollo sicológico al margen del contexto histórico- 
cultural en el que está inmerso, lo que trae consigo una serie de instrumentos y prácticas sociales históricamente determinados y organizados (Rogoff, 1993).

Además esta corriente de pensamiento describe las relaciones entre dos áreas: la psicología y la educación (Vigotsky, 1926). Para este autor, las relaciones entre estas disciplinas son de influencia mutua, debido a que integran ejes como el aprendizaje, el desarrollo sicológico, la educación y la cultura. De ahí que Vigotsky se interesara por el uso de principios y normas sicológicas en el campo pedagógico (Hernández, 2001).

Abundando en este marco teórico, señalamos que los procesos cognitivos en el desarrollo dependen de la interacción cooperadora entre un individuo y otro con más conocimientos y apropiación de la cultura, que le ayuda a adquirir los instrumentos simbólicos necesarios para el aprendizaje; es así, como surge el concepto de la ZDP que se define como: «La distancia entre el nivel real de desarrollo, determinada por la capacidad de resolver independientemente un problema, y el nivel de desarrollo potencial, determinado a través de la resolución de un problema bajo la guía de un adulto o en colaboración con otro compañero más capaz» (Vigotsky, 1979, p. 133).

En línea con dicho autor, Wertsch (1993) señala que el alumno reconstruye los saberes, pero no de forma individual, puesto que ocurren procesos complejos en los que se entretejen procesos de construcción personal y procesos auténticos de co-construcción en colaboración con los otros que intervinieron, de alguna manera, en este proceso.

Por otra parte, Hernández (2001) manifiesta que el ser humano se desarrolla en la medida en que se apropia de una serie de instrumentos de índole sociocultural y cuando participa en tales prácticas y relaciones sociales con otros que saben más que él sobre esos instrumentos y prácticas.

Los instrumentos culturales son aquellos que intermedian el crecimiento cultural $-\mathrm{y}$ por ende, el aprendizaje- de una persona situada en un contexto específico. Dichos instrumentos se consideran mediadores entre el sujeto y el objeto y se clasifican en dos formas: los instrumentos materiales y los instrumentos sicológicos.

Los instrumentos materiales están orientados externamente, destinados a ser usados para interactuar con la realidad física y provocar cambios en ella. Tienen una influencia indirecta en los procesos sicológicos humanos.

Los instrumentos psicológicos, por su parte, se orientan internamente y median entre los procesos sicológicos de los seres humanos para influir, primeramente, en los demás y, luego, en uno mismo (Hernández, 2011). 
En el campo de la educación, el interés se centra en comprender cómo el conocimiento se comparte y gestiona entre profesores y alumnos en el proceso educativo. En esta participación y gestión intervienen instrumentos culturales tales como libros, calculadoras, pizarras, proyectores, televisores; tecnologías de la información y la comunicación (TIC); reproductores de audio y vídeo, entre otros.

Aunados a los anteriores, los instrumentos culturales sicológicos, también, intermedian este proceso de colaboración e interacción entre los participantes del hecho educativo; así, encontramos en este tipo de instrumentos los discursos educativos, las estrategias de enseñanza, las estrategias de aprendizaje, el lenguaje oral, el lenguaje escrito, el código o lenguaje que se manejan en los medios visuales, auditivos y digitales, entre otros.

En función a estas premisas, se conforma el sustento teórico para las actividades y acciones constituidas en el proceso de las tutorías abordadas en este estudio.

\section{Metodología}

El diseño metodológico de la investigación parte de los supuestos de los estudios interpretativos porque se requiere comprender un fenómeno educativo mediante el análisis de las percepciones e interpretaciones de los participantes que intervienen en la acción pedagógica. Con base en esta comprensión, se pretende llegar a la captación de las relaciones internas y profundas, indagando en la intencionalidad de las acciones y en las percepciones de los sujetos (Colás y Buendía, 1998). De igual manera, se establece un diseño metodológico debido a que se toman decisiones a lo largo de todo el proceso de investigación y sobre todas las fases o pasos que conlleva dicho proceso (Valles, 1999).

\subsection{Método}

El método seleccionado para la investigación es el estudio de casos, ya que permite estudiar la particularidad y la complejidad de un caso singular, para llegar a comprender su actividad en circunstancias importantes (Stake, 2007), como lo es el proceso de acción tutorial en la cotidianeidad de una institución de educación superior mexicana, sin pretender generalizar los hallazgos; más bien, se trata de particularizar sobre el caso para conocerlo a profundidad, puesto que la generalización se promueve en la medida en que el investigador "intenta proporcionar la información elaborada sobre la cual los lectores deciden el grado en el que el caso investigado es similar al suyo" (Stake, 1985, pág. 280).

En especial, se trata de un estudio de caso instrumental porque está enfocado al análisis de casos como instrumentos para evaluar y mejorar procesos específicos relacionados con el caso analizado (Stake, 2007), es decir, los hallazgos generados por las significaciones otorgadas por los participantes 
del fenómeno educativo servirán para mejorar la implementación y el desarrollo del programa de acción tutorial en la carrera en referencia.

\subsection{Objetivo}

El objetivo general del estudio consiste en explicar la experiencia del tutor y el tutorado en el transitar del proceso formativo en la Licenciatura en Sistemas Computacionales de la Universidad Autónoma de Chiapas, México. Por tanto, se procede ante la realidad que se pretende encarar, la cual se constituye como una situación multicausal, multidimensional y por ende compleja, al comprender e interpretar de viva voz de los participantes los significados que le atribuyen a la tutoría en el proceso de enseñanza y aprendizaje, en la que los involucrados fungen diversos roles y situaciones contextuales en el devenir del programa educativo señalado.

\subsection{Muestra}

La selección de los participantes se efectuó considerando los presupuestos del muestreo basado en criterios, el cual señala que en la muestra intencional se elige una serie de criterios que son necesarios o muy convenientes para los fines que persigue la investigación. En consecuencia, se procura que dicha muestra represente lo mejor posible los subgrupos naturales, que sea una muestra comprensiva, tomando en cuenta los casos de mayor representatividad y practicidad (Martínez, 2006). De igual modo, el muestreo basado en criterios establece que "requiere únicamente que el investigador confeccione un listado de los atributos esenciales que debe poseer la unidad seleccionada para, a continuación, localizar en el mundo real alguna que se ajuste a ellos" (Goetz y LeCompte, 1988, pág. 98).

Para esta investigación, se establecieron los siguientes criterios:

1. Profesores que funjan como tutores en la carrera, no importando su estatus de contratación.

2. Profesores que se encuentren anuentes en colaborar con la investigación.

3. Estudiantes que hayan recibido tutoría de forma continua en el transitar por el programa educativo.

Una vez establecidos estos criterios, se decidió trabajar con séis profesores que poseen el estatus de tiempo completo, medio tiempo y asignatura de la Licenciatura en Sistemas Computacionales y 31 tutorados de los profesores tutores participantes; dichos tutores y tutorados corresponden a los semestres de primero, cuarto, sexto y noveno de la carrera.

Esta selección se realizó considerando el perfil establecido como necesario para este estudio y la anuencia de los actores educativos de ser partícipes de nuestra investigación, facilitando el acceso a sus tutorados para la aplicación de técnicas de recolección y análisis de información. 


\subsection{Técnicas}

La realidad que se estudia es compleja, por lo que se deben seleccionar técnicas adecuadas para hacer acopio de la información; mediante la puesta en práctica de las técnicas seleccionadas se profundizará en el objeto de estudio de esta investigación.

Las técnicas de recolección y el tratamiento analítico de la información corresponden a la entrevista en profundidad, el grupo focal y la revisión de documentos. Igualmente, las técnicas de análisis aluden a la suma categórica, la correspondencia y modelos y las generalizaciones naturalistas.

La entrevista en profundidad permite obtener las descripciones e interpretaciones que los agentes de la educación poseen de dicha realidad, siendo la entrevista un cauce principal para llegar al abordaje de las realidades múltiples (Stake, 2007).

Por consiguiente, se elaboró una guía de entrevista para la aplicación de esta técnica a los profesores tutores con tópicos relacionados con la valoración de la tutoría en el proceso educativo, las funciones realizadas por el tutor, la organización de las actividades tutorales, la capacitación brindada para ejercer el rol, el conocimiento sobre el programa institucional de tutoría y las herramientas utilizadas para realizar dicho proceso. En total, el instrumento se conformó con 15 preguntas que abarcan los siete ejes expuestos.

Las entrevistas en profundidad se efectuaron en tres etapas: inicio, intermedio y cierre del ciclo escolar enero-junio de 2017. De esta manera, las entrevistas se llevaron a cabo en los meses de enero, abril, mayo, junio y julio del mismo año.

La segunda técnica utilizada fue el grupo focal, debido a que, según Stewart y Shamdasani (1990, pág. 16-19):

La situación de grupo hace que las respuestas o intervenciones surjan como reacción a las respuestas o intervenciones de otros miembros presentes en la reunión. Se trata del efecto de sinergia, provocado por el propio escenario grupal y que resulta en la producción de información que pudiera no producirse en las entrevistas individuales entre entrevistador y entrevistado.

Así, se aplica esta técnica a los tutorados con la que se recaba su opinión; para ello, se elaboró una guía que aborda los tópicos sobre la valoración de las tutorías recibidas, el conocimiento que poseen sobre el Programa de acción tutoral implementado en la institución, los tipos de tutorías que han necesitado y recibido, el historial de asesorías durante su formación y la incidencia que estas tutorías han tenido en su aprendizaje. 
Debido a lo cual, se conformaron cuatro grupos focales con estudiantes de los semestres referidos de la Licenciatura en Sistemas Computacionales de la UNACH; tres de estos grupos se formaron por ocho estudiantes y un solo grupo se compuso por siete. Para el desarrollo del trabajo, se realizaron seis sesiones grupales en las mismas fechas en las que se efectuaron las entrevistas a los tutores. Las sesiones de grupo se llevaron a cabo como se especifica en la Tabla 1.

\begin{tabular}{|l|l|l|l|}
\hline$N^{0}$. sesión & Periodo & Semestre & Duración \\
\hline 1 & Inicio del ciclo escolar & Primero & 80 minutos \\
\hline 2 & Intermedio del ciclo escolar & Segundo & 60 minutos \\
\hline 3 & Intermedio del ciclo escolar & Cuarto & 60 minutos \\
\hline 4 & Intermedio del ciclo escolar & Sexto & 60 minutos \\
\hline 5 & Final del ciclo escolar & Noveno & 60 minutos \\
\hline 6 & Final del ciclo escolar & Primero & 60 minutos \\
\hline
\end{tabular}

Tabla 1. Realización de las sesiones de grupo focal con estudiantes Fuente: elaboración propia (2017)

Asimismo, para la descripción de los significados de los actos que acontecen en los espacios físicos, los contextos históricos, culturales o estéticos en los que se desenvuelven los actores del fenómeno educativo, aspectos indispensables para alcanzar las significaciones, se utilizó la descripción de los contextos en los que se lleva a cabo la actividad investigadora.

La última técnica aplicada para la recolección de datos ha sido la revisión documental. Revisamos y analizamos investigaciones acerca de la implementación de las tutorías en el contexto educativo superior que datan del año 2012 al 2017, los diversos formatos de tutoría que utilizan los tutores en el desempeño de su función (formato de entrevista, análisis FODA, cuestionario de hábitos de estudio, cuestionario para detección de niveles de asertividad, índice de autoestima y cuestionario de estilos de aprendizaje); la carpeta de evidencias que cada tutor elabora de los tutorados asignados; y el sistema de información computacional desarrollado específicamente para el seguimiento de las tutorías en la institución.

En relación con el análisis de la información, se emplearon las técnicas de suma categórica, correspondencia y modelos y generalizaciones naturalistas. Mediante la suma categórica se busca un conjunto de ejemplos y de la suma de ellos emergen significados relevantes sobre el objeto de estudio; congruente con este análisis, la correspondencia y modelo permite la búsqueda del significado 
a través de modelos, de consistencia en determinadas condiciones, a lo que en investigación con estudio de caso se denomina correspondencia (Stake, 2007).

En definitiva, las generalizaciones naturalistas hacen posible la explicación narrativa considerando el tiempo, el lugar y los sujetos de estudio que intervienen en el fenómeno educativo analizado.

De tal manera, por medio de la triangulación múltiple efectuada con las estrategias de recopilación y análisis de la información establecida en el diseño metodológico, se explican las comprensiones otorgadas por los participantes del proceso de tutoría en el transitar por la carrera de Sistemas Computacionales. La figura 1 esquematiza lo explicado:

Recopilación y análisis de la información

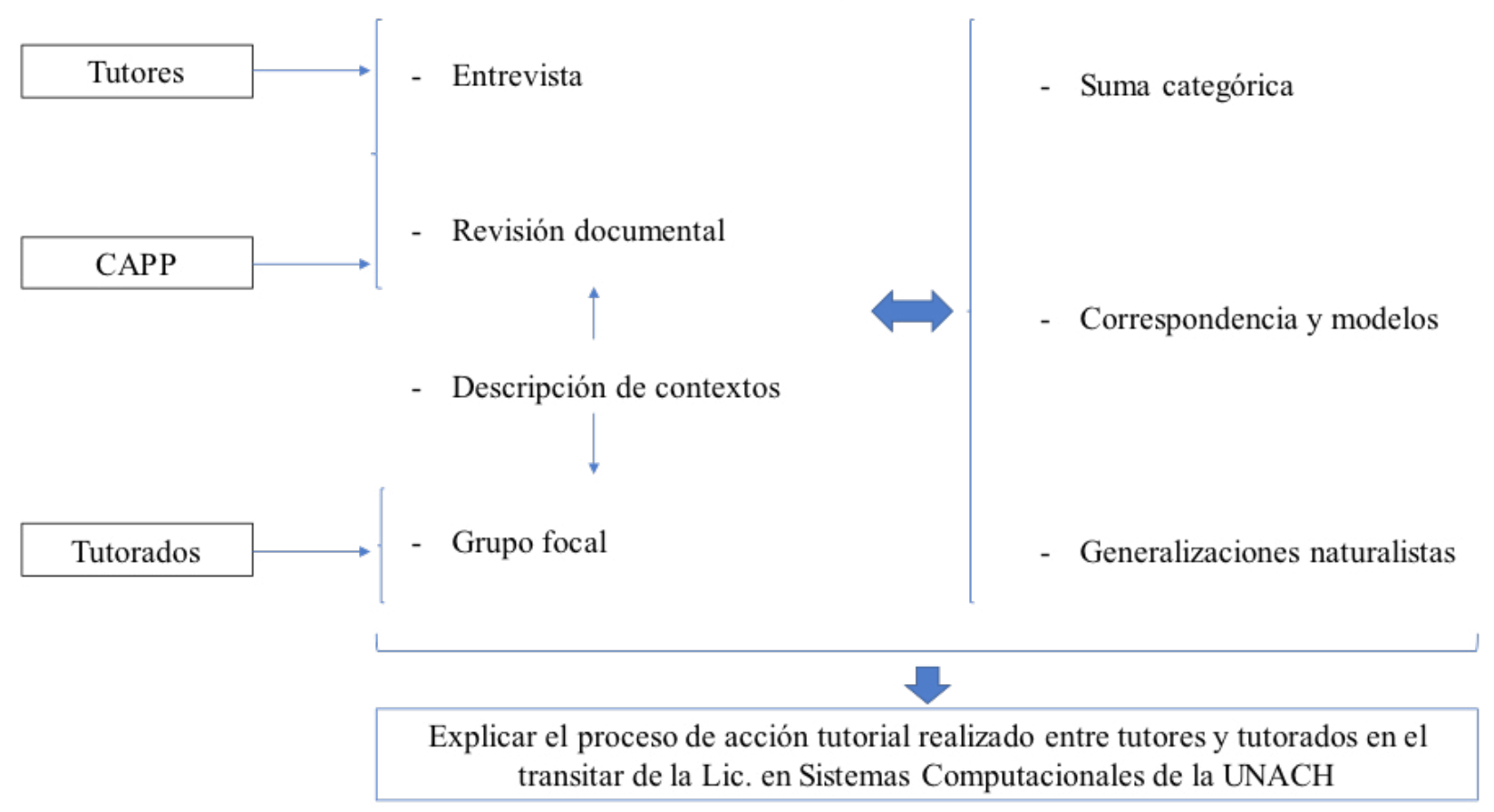

Figura 1. Diseño metodológico mediante el estudio de casos

Fuente: elaboración propia (2017)

\section{Resultados}

Derivado del análisis efectuado de nuestro trabajo de campo, emergieron tres categorías que, en su conjunto, explican el objeto de investigación. Dichas categorías se detallan en la Tabla 2. 


\begin{tabular}{|c|c|c|}
\hline \multicolumn{2}{|r|}{ Categoría } & Propiedades \\
\hline 1) & $\begin{array}{l}\text { Valoración de las tutorías por } \\
\text { parte del docente tutor }\end{array}$ & $\begin{array}{ll}\text { - } & \text { Asignación de tutorados } \\
\text { - } & \text { Conceptualización de la tutoría } \\
\text { - } & \text { Funciones del tutor } \\
\text { - } & \text { Limitaciones del tutor } \\
\text { - } & \text { Separación de la función docente y la función tutorial } \\
\text { - } & \text { Autovaloración del desempeño de la función tutorial }\end{array}$ \\
\hline 2) & $\begin{array}{l}\text { Valoración de las tutorías por } \\
\text { parte de los estudiantes }\end{array}$ & $\begin{array}{l}\text { - } \\
\text { - } \\
\text { - } \quad \text { Defiginición de la tutoría } \\
\text { - } \quad \text { Valoración del tutoriones de la tutoría en el desempeño escolar } \\
\text { - }\end{array}$ \\
\hline 3) & $\begin{array}{l}\text { Estrategias para la } \\
\text { implementación del Programa } \\
\text { de Acción Tutorial (PAT) en } \\
\text { la Licenciatura en Sistemas } \\
\text { Computacionales }\end{array}$ & $\begin{array}{l}\text { - Formación para ejercer el rol de tutor (manejo de grupo, manejo de } \\
\text { personas conflictivas, orientación sobre las preferencias sexuales, } \\
\text { detección y manejo de adicciones y uso de la plataforma tecnológica) } \\
\text { - Actividades y tareas para ejercer la tutoría }\end{array}$ \\
\hline
\end{tabular}

Tabla 2. Categorías y propiedades de las comprensiones e interpretaciones del tutor y el tutorado en el transitar por el programa educativo LSC. Fuente: elaboración propia (2017)

\subsection{Valoración de las tutorías por parte del docente tutor}

Esta primera categoría define el concepto de la tutoría, la implementación del PAT en la carrera y las funciones que lleva a cabo el docente en su calidad de tutor. De este modo, identificamos que los profesores definen la tutoría como una orientación integral que considera el ámbito personal, académico y profesional de los estudiantes para que estos inicien, transiten y finalicen sus estudios en el programa educativo.

En este sentido, se manifiesta que "la tutoría tiene la finalidad de orientar al estudiante en la cuestión académica, en aspectos psico y socioemocionales del estudiante durante su permanencia, porque van ellos evolucionando y creciendo; y en situaciones, también, sociales como puede ser la generación de un empleo" (S., comunicación personal mediante entrevista, 31 de enero de 2017). "La tutoría es el acompañamiento de los estudiantes en las diversas dificultades que tienen en su trayectoria de formación académica y en aquellas actividades que han culminado con éxito; acompañarlos y reforzar lo necesario para que tengan mejor logro académico" (J., comunicación personal mediante entrevista, 07 de julio de 2017).

Asimismo, la tutoría se debe enfocar en "la parte humana del estudiante, y los tutores debemos contar con la suficiente sensibilidad humana para generar confianza en los alumnos y poderlos orientar y ayudar" (K., comunicación personal mediante entrevista, 27 de junio de 2017). 
De acuerdo con lo planteado, los tutores participantes exponen que la tutoría es una actividad adicional a su quehacer docente, que se centra en la orientación de los estudiantes para solventar aquellas situaciones sociales, económicas y afectivas que permean su desempeño escolar; mediante la tutoría se guía al tutorado en el devenir de sus estudios universitarios, con el objetivo de que este culmine exitosamente su proceso de formación.

En relación con la implementación del Programa de Acción Tutorial en la carrera, los docentes consideran que la institución y, particularmente, la Facultad en la que se encuentra inscrita la carrera han hecho una gran labor en la ejecución del programa de tutorías. Al respecto se señala que "la Facultad sí se ha preocupado por la implementación de la tutoría, debido principalmente a los procesos de acreditación de las carreras" (S., comunicación personal mediante entrevista, 14 de abril de 2017).

Los profesores valoran el PAT como un programa completo, que integra los tres momentos significativos en la formación de los estudiantes: inicio, permanencia y culminación, en el que se cuenta con el apoyo de los directivos, el coordinador de carrera, la coordinadora de academia y el responsable del Centro Psicopedagógico de la Facultad. No obstante, lo profesores señalan que el programa de tutorías no se ha consolidado en las carreras que se ofertan, incluida la Licenciatura en Sistemas Computacionales, pues no hay una aplicación real de las tutorías, es decir, existe asignación de tutorados a profesores que deben desempeñarse como tutores durante un semestre, pero no hay un desempeño efectivo de las tutorías.

Respecto a las funciones del tutor, los docentes concuerdan en que se deben llevar a cabo las siguientes:

1. Realizar una inducción del programa de tutorías con los estudiantes.

2. Dar a conocer a los estudiantes los lineamientos institucionales.

3. Realizar un diagnóstico personal y académico de los tutorados.

4. Ayudar en la integración de los estudiantes a la dinámica institucional.

5. Definir el alcance de su papel como tutor.

6. Explicar el plan de estudios de la carrera.

7. Realizar actividades para la integración grupal.

8. Establecer un calendario de tutorías.

9. Explicar los servicios académicos institucionales proporcionados en beneficio de la formación de los estudiantes (becas, integración al seguro social, gestiones escolares, entre otros). 
10. Orientar sobre temas vocacionales.

11. Proporcionar estrategias para mejorar los hábitos de estudio

12. Gestionar cursos académicos en asignaturas que se le dificulten a los estudiantes y en aquellos temas de interés del mismo.

13. Canalizar a los tutorados que requieran la atención de especialistas de otras áreas o instituciones.

14. Registrar el número y el tipo de tutorías otorgadas en la agenda del tutor y en el sistema de cómputo.

15. Conformar una carpeta de tutorías con las evidencias de la atención tutorial (formatos e informes).

16. Realizar el cierre de las actividades de tutorías con los tutorados asignados.

De esta manera, los profesores consideran que se abarcan los procesos y las actividades que el alumnado debe afrontar durante su formación profesional en la institución.

En relación con la modalidad de las tutorías, estas se llevan a cabo principalmente de dos maneras: grupal e individual, en la que predominan las sesiones grupales sobre las individuales. Esto no significa que los tutores no realizan tutorías de forma individual; más bien, representa que los estudiantes solventan la mayor parte de sus dudas durante el tiempo dedicado a la tutoría en el aula, y son menos los estudiantes que recurren a una cita con el tutor, en un horario distinto a clases, para abordar problemáticas de orden económico, afectivo, laboral, psicosocial, de preferencias sexuales y académico.

\subsection{Valoración de las tutorías por parte de los estudiantes}

La segunda categoría surgida del trabajo de campo explica la concepción que tiene el estudiantes sobre las tutorías que le han concedido, su percepción sobre el Programa tutorial institucional y las implicaciones de contar con este tipo de atención en su proceso educativo.

Los estudiantes reconocen que han contado con orientación tutorial desde el inicio de su carrera, la que se traduce en la impartición de cursos de motivación, cursos orientados a las asignaturas de su carrera, cursos sobre salud sexual y adicciones, y cursos de emprendimiento. Al respecto se señala que "las tutorías son formas de motivarnos, apoyarnos en clase, es un camino para ayudarnos a seguir adelante en nuestros estudios" (X., comunicación personal mediante grupo focalizado, 04 de mayo de 2017); "nos ayudan para tener un poco más de conocimientos sobre cualquier tema, contribuyen en nuestro aprendizaje" (Y., comunicación personal mediante grupo focalizado, 04 de mayo de 2017). 
De acuerdo con lo expuesto por los estudiantes, se considera que las tutorías sirven, principalmente, para hacer frente a situaciones académicas que permitan una mejor comprensión e interiorización de las temáticas que más les dificultan, como, por ejemplo, las matemáticas y las materias de programación. Aunque, también, se manifiesta que están orientadas a resaltar temas motivacionales y revertir algunos factores que pueden influenciar negativamente en el rendimiento académico.

Por otra parte, los estudiantes no conocen a detalle el Programa Institucional de Tutorías (PAT), que se ha implementado desde el año 2013 en la Facultad, pero reconocen que el profesorado funge, además, con el papel de tutor, puesto que desde el principio de la carrera han recibido tutorías, más grupales que individuales, en el transcurso de su formación. Esto contradice, en parte, lo manifestado por los profesores tutores los cuales señalan que se realiza una inducción sobre dicho programa desde el inicio de la carrera; además de los informes estadísticos presentados por las instancias responsables de la realización de las tutorías, en todas sus modalidades.

Sobre las implicaciones del PAT en su formación académica, los estudiantes se pronuncian a favor de la integración de las tutorías a su proceso de aprendizaje, subrayando que "son mecanismos que nos proporcionan los tutores para ayudarnos a aprobar las materias, desde cursos, explicaciones individuales sobre los temas que no entendemos, consejos personales y escolares, asesorías para mejorar nuestros hábitos de estudios y todo lo relacionado con la aprobación de todas las materias del semestre" (A., comunicación personal mediante grupo focalizado, 19 de mayo de 2017).

"El tutor nos ayuda porque es la persona que te asesora, no solo en su materia, sino en todas las demás para ver cómo vas, o si vas mal en alguna materia te puede apoyar con clases, clases por aparte para que sepas más" (D., comunicación personal mediante grupo focalizado, 19 de mayo de 2017); "el tutor se enfoca en el alumno de forma integral y monitoriza nuestro progreso en la escuela" (H., comunicación personal mediante grupo focalizado, 19 de mayo de 2017).

Consecuentemente, los tutores resultaron evaluados de forma muy satisfactoria, con una etiqueta de excelentes o muy buenos debido a la atención y seguimiento constante de las problemáticas expuestas por sus tutorados; sin embargo, es importante mencionar que es necesario que los tutores dispongan de más tiempo de atención para sus tutorados.

Por estas razones, se manifiesta que las tutorías han beneficiado su permanencia en la carrera puesto que mediante ellas se les ha motivado de forma constante, proporcionándoles alternativas para superar los obstáculos que se van presentando en el transcurso de sus estudios, es decir, la implementación del programa de tutorías ha servido para revertir la deserción escolar, tal como lo manifiestan Hernández, Martínez y Carranza (2013) en su investigación. 


\subsection{Estrategias para la implementación del Programa de Acción Tutorial (PAT)}

La tercera categoría surgida del análisis aborda las actividades y las tareas que resultan necesarias llevar a cabo para aplicar de forma efectiva el programa académico en referencia. En este aspecto, se identifican 10 actividades y tareas que deben efectuarse antes, durante y después de ejercer la tutoría, tal como se muestra en la Tabla 3.

\begin{tabular}{|c|c|c|c|}
\hline Actividades y tareas & Antes & Durante & Después \\
\hline Inducción del PAT al profesorado & $x$ & & \\
\hline $\begin{array}{l}\text { Capacitación sobre el manejo del sistema de información para registrar las } \\
\text { tutorías }\end{array}$ & $x$ & & \\
\hline Conocimiento sobre las funciones del tutor, su alcance y ámbito de trabajo & $X$ & & \\
\hline Capacitación docente sobre integración grupal & $x$ & $x$ & \\
\hline Capacitación docente sobre detección de adicciones & $X$ & $X$ & \\
\hline $\begin{array}{l}\text { Capacitación docente sobre manejo de las preferencias sexuales de los es- } \\
\text { tudiantes }\end{array}$ & $x$ & $x$ & \\
\hline Curso sobre manejo de emociones y personas conflictivas & 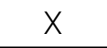 & $x$ & \\
\hline Accesibilidad y disponibilidad de horario para ejercer la tutoría & & $x$ & \\
\hline $\begin{array}{l}\text { Conocimiento de los servicios proporcionados por las áreas institucionales } \\
\text { e instancias externas para la canalización de los estudiantes con prob- } \\
\text { lemáticas psicopedagógicas, socioemocionales y salud. }\end{array}$ & $X$ & $X$ & $x$ \\
\hline $\begin{array}{l}\text { Coordinación sistemática y continua con el Centro de Apoyo Psicopedagógi- } \\
\text { co de la Facultad }\end{array}$ & $x$ & $x$ & $X$ \\
\hline
\end{tabular}

Tabla 3. Estrategias para ejercer la tutoría en la Licenciatura en Sistemas Computacionales Fuente: elaboración propia (2017)

Estas actividades y tareas surgidas en la investigación aplican, particularmente, en la implementación del Programa de Acción Tutorial en la carrera en la que se sitúa el contexto de la investigación.

\section{Discusión}

La discusión de los resultados obtenidos en esta investigación se exponen considerando datos de referencia ofrecidos por autores que han realizados estudios en esta temática, señalando aspectos comunes y divergentes de los resultados obtenidos.

Si bien la tutoría ha sido un proceso que ha beneficiado a los estudiantes de la carrera en la que se ha llevado a cabo la investigación, el propósito establecido permite dar una explicación de aspectos resultantes del proceso investigativo. Es necesario puntualizar que algunas de las problemáticas identificadas no se atienden efectivamente con la implementación del programa institucional de tutorías. Ejemplo de ello es la deficiencia académica que presentan los estudiantes en asignaturas como matemáticas, algoritmos y programación, que son a su vez las materias que más se le dificulta y son causa de baja escolar. 
En este sentido, es importante señalar que las deficiencias académicas detectadas en los estudiantes, muchas de ellas acarreadas del nivel medio superior, no son, ni serán, resarcidas mediante un programa de tutoría universitaria. Esta es una problemática distinta que se presenta de forma constante con el paso de un nivel educativo a otro, en este caso, del nivel medio-superior al superior.

De acuerdo con los hallazgos que se derivan de este estudio, y a la vista de investigaciones como la de Álvarez y Bisquerra (2010), en cuanto a darle un papel relevante a los roles de quienes participan en los procesos de tutoría, hay un acuerdo común en el rol que debe cumplir el tutor, señalando lineamientos que deben realizar cuando se desarrollan las tutorías, especialmente, tal y como estos autores abordan, la experiencia en la que se persigue el desarrollo de emociones interculturales en el aula.

Un elemento en particular que se evidencia en nuestra investigación es que aporta características de la prevalencia de la vulnerable situación económica de los alumnos, lo que representa otra de las causas en la deserción escolar. En este caso, las tutorías proporcionan una orientación continua sobre las becas institucionales a las que los estudiantes pueden acceder y se les guía para la obtención de empleo. Sin embargo, muchas veces la situación socioeconómica del estudiante sobrepasa los esfuerzos hechos por los tutores y por el programa en sí, no permitiendo decrementar de manera significativa la deserción escolar en la carrera, que ha pasado de contar con 739 alumnos matriculados (UNACH, 2010) a 422 (UNACH, 2015) en cinco años.

Por ello la importancia de realizar este tipo de investigaciones, que den luz sobre las estrategias institucionales implementadas para revertir la problemática existente, de viva voz de los participantes del fenómeno educativo examinado. En este sentido, se concuerda con Noh (2013) en que este tipo de investigaciones permite exponer problemáticas que vive el estudiante relacionados con la deserción, rezago educativo, abandono de los estudios y mejora de eficiencia terminal. Disponer de un panorama claro, puede llevar al establecimiento de acciones en las universidades para solventar este problema y proponer nuevas estrategias de trabajo sobre la permanencia de estudiantes en el nivel universitario.

Un aspecto divergente es el de Henríquez (2008), que analiza la interacción entre docentes y estudiantes, principalmente. Sobre la calidad de los proyectos y la investigación que se expone en su documento, argumenta una explicación que consiste en detallar las experiencias que se establecen entre el tutor y tutorado a lo largo del proceso de formación, en la carrera y universidad en estudio.

Por último, se manifiesta que el PAT es un programa completo, congruente con las políticas educativas del nivel superior en este rubro y posee el apoyo institucional para su cumplimiento. No obstante, la clave para la efectiva implementación de dicho programa es el compromiso docente para ejercer este otro rol, el de tutor, que establece una práctica real de estrategias en beneficio evidente 
de la formación de los estudiantes; de otro modo, el programa corre peligro de convertirse en una simulación burocrática, es decir, en la mera realización de actividades que el docente y la institución deben acreditar para alcanzar un buen desempeño académico.

En particular esta investigación difiere con los resultados de otros estudios sobre tutorías, debido al tipo de abordaje, mediante el estudio de caso. Las tutorías se han visto así como una posición de desarrollo integral para el logro de las metas educativas, en el contexto en estudio.

\section{Conclusiones}

Las principales conclusiones del estudio permiten señalar que, de la investigación se derivan hallazgos que admiten dar una explicación de las experiencias de formación tanto de los tutores como de los tutorados en la Licenciatura en Sistemas Computacionales de la Universidad Autónoma de Chiapas, México. Los hallazgos relevantes hasta este momento del desarrollo investigativo están relacionados con la posición que tienen las tutorías como una opción educativa integral, pues se toma en cuenta los distintas acciones que tienen que ver con la persona, principalmente desde una posición académica, que le permita al tutorado alcanzar sus estudios de licenciatura, además de un crecimiento psicológico, social e integral.

También es relevante atestiguar que la orientación del programa de tutorías permite reconocer que, mediante su desarrollo, se logra un trabajo que motiva a los tutorados a obtener orientaciones sobre las diferentes asignaturas, apoyos en las clases y actividades que les facilitan culminar con los estudios. Asimismo dichas tutorías han permitido a los tutorados obtener información de acceso a becas institucionales, gestiones académicas y administrativas inter e intra institucional y opciones laborales, aspectos que les brinda posibilidades de alcanzar mejores oportunidades personales.

El alcance que ha tenido el tutorado en el desempeño escolar, al ser partícipe de este proceso educativo, es significativo, ya que los mismos tutores señalan que trabajan para ofrecer oportunidades a los tutorados y que estos puedan solventar las diferentes situaciones que les aquejan, desde lo social, lo económico y lo afectivo, lo que puede incidir en su desempeño en el proceso formativo. Igualmente, trabajan motivando a que los tutorados mantengan abiertas oportunidades educativas del nivel superior y alcancen sus metas en el ámbito de la educación.

Otro de los hallazgos que cobra importancia en esta investigación es que los tutorados indican que los tutores son excelentes o muy buenos, no solo por el trabajo relacionado con los estudios, sino porque se abordan distintas temáticas que les fortalecen en diversas áreas. Y manifiestan que es necesario que se incremente la cantidad de horas de atención de los tutores.

La información permite señalar que es indispensable darle seguimiento a los tres momentos de 
ejecución del desarrollo de la tutoría, para que se obtengan mejores resultados en el tutorado que participa de ellas. Por lo que se recomienda el uso de las actividades y tareas establecidas, según las recomendaciones y necesidades de los tutorados.

En suma, si bien el programa de tutorías ha beneficiado a los tutorados en distintas formas, aún quedan acciones por implementar para que los problemas de esta población se resuelvan positivamente, generando una solvencia de las diferentes realidades de la población, específicamente, en cuanto a la deserción, vulnerabilidad y situaciones económicas.

\section{Referencias}

Alcolea, G. y Pérez, M. (2012). Un paso más allá en la planificación docente universitaria: el plan de acción tutorial. Estudios sobre el Mensaje Periodístico, 18, 45-55. doi:https://doi.org/10.5209/rev_ ESMP.2012.v18.40889

Alvarado, V. y R. (2006). Los aspectos cualitativos de la tutoría en educación superior. Matices: Revista de Posgrado, 1(1), 37-47.

Álvarez, M. y Bisquerra, R. (Coord.) (2010). Manual de orientación y tutoría. Barcelona: Wolters Kluwer. Edición en CD nº. 41.

Asociación Nacional de Universidades e Instituciones de Educación Superior. (2001). Programas institucionales de tutoría. México: ANUIES.

Colás, M. y Buendía, L. (1998). Investigación Educativa. Sevilla: Ediciones ALFAR.

Goetz, J. \& LeCompte, M. (1988). Etnografía y diseño cualitativo en investigación educativa. Madrid: Ediciones Morata, S.A.

Henríquez, G. (2008). La tutoría electrónica aplicada en los proyectos de investigación en salud RIED, 12(1), 79-93.

Hernández, G. (2001). Paradigmas en psicología de la educación. Ecuador: Paidós.

Hernández, G. (2011). Miradas constructivistas en psicología de la educación. México: Paidós.

Hernández Y., Martínez, J. y Carranza, C. (2013). Programa Institucional de Tutorías; Una estrategia para elevar el nivel de aprovechamiento en una institución educativa. Acta universitaria, 1(23), 31-36.

Herrera, L. (2011). Orientación, tutoría y mentorización en Educación Superior: Una labor destinada tanto al alumnado como al profesorado universitario. Dedica. Revista de educação e humanidades, 1 , 425-452. 
Kozulin, A. (1994). La psicología de Vigotsky. Madrid: Alianza.

Martínez, M. (2006). Ciencia y Arte en la metodología cualitativa: Métodos hermenéuticos, métodos fenomenológicos, métodos etnográficos. México: Trillas.

Narro, J. y Arredondo, M. (2013). La tutoría. Un proceso fundamental en la formación de los estudiantes universitarios. Perfiles Educativos, 141(35), 132-151.

Organización de las Naciones Unidas para la Educación, la Ciencia y la Cultura (UNESCO). (1998). Declaración Mundial sobre la Educación Superior en el siglo XXI: Visión

Noh, R. (2013) Las TIC como herramientas para tutorías académicas. Pistas Educativas, 101, 134-143.

Rodríguez, N. y Sierra, E. (2014). Reflexiones pedagógicas sobre la tutoría académica en educación superior. Revista Salud Bosque, 1 (4), 29-36.

Rodríguez, C., Calvo, A. y Haya, I. (2015). La tutoría académica en la evaluación superior. Una investigación a partir de entrevistas y grupos de discusión en la Universidad de Cantabria (España). Revista Complutense de Educación, 2(26), 467-481.

Rogoff, B. (1993). Aprendices del pensamiento. Desarrollo cognitivo en el contexto social. España: Paidós.

Stake, R. (1985). Case Study. En Nisbet, J. (Ed) World Yearbook of Education 1985. Research, Policy and Practice. London: Kogan Page.

Stake, R. (2007). Investigación con estudio de casos. Madrid: Ediciones Morata, S. L.

Stewart, D. y Shamdasani, P. (1990). Focus groups. Theory and practice. London: Sage.

Torres, J. (2013). Relación de tutoría y promoción del desarrollo de habitus científicos en estudiantes de doctorado en educación. Acercamiento a un caso. Perfiles Educativos, 140(35), 8-27. doi:https:// doi.org/10.1016/S0185-2698(13)71819-1

UNACH. (2010). Anuario estadístico 2010. Chiapas: UNACH.

UNACH. (2015). Anuario estadístico 2015. Chiapas: UNACH.

Valles, S. (1999). Técnicas cualitativas de investigación social. Madrid: Editorial Síntesis, S. A.

Vigotsky, L. (1926). Los métodos de investigación reflexológicos y psicológicos, en L.S. Vigotsky (1991), Obras escogidas, vol. I. Madrid: Visor.

Vigotsky, L. (1979). El desarrollo de las funciones psicológicas superiores. Barcelona: Grijalbo. 
Wertsch, J. (1993). Voces de la mente. Un enfoque sociocultural para el estudio de la acción mediada. Madrid: Visor. 